\title{
Direct distance determination using parallax: Techniques, promises and limitations
}

\author{
Lennart Lindegren
}

Lund Observatory, Lund University, Box 43, SE-22100 Lund, Sweden

email: lennart.lindegren@astro.lu.se

\begin{abstract}
Determination of trigonometric parallaxes is one of the very few direct methods available for measuring cosmic distances, and the only one capable of reaching beyond the Solar System. It is fundamental both to determine the intrinsic properties of astrophysical objects and as the first step on the cosmic distance ladder. Steady improvements of instruments and techniques have extended the applicability of the parallax method to ever greater distances. However, it is not correct to say that the method is independent of physical assumptions. This review covers ground-based optical techniques, from Bessel's time to the present, as well as space observations and radio interferometry. Some emphasis is put on the physical limitations of the method, in particular its sensitivity to source structure and photocentric variability on different timescales.
\end{abstract}

Keywords. astrometry, stars: distances, solar neighborhood, Galaxy: general

\section{What is parallax?}

If the stars were fixed in space, relative to the Sun, each would appear to describe a small ellipse as seen by an observer on the Earth. This parallax ellipse mirrors the Earth's orbit as seen from the star: the semi-major axis equals the angle subtended by the astronomical unit (au) at the distance of the star: that is the parallax $\varpi$; the axis ratio equals $\sin \beta$, where $\beta$ is the ecliptic latitude of the star; and the phase of the parallax motion varies with the ecliptic longitude of the star. Real stars are in motion, however, which usually can be modelled quite accurately over several years as a constant angular velocity or proper motion, $\mu$. Combining the two effects, it is found that the variation in any angular coordinate $\theta$ (such as $\alpha$ or $\delta$ ) can be modelled as

$$
\theta(t)=\theta_{0}+\left(t-t_{0}\right) \mu+F_{\theta}(t, \alpha, \delta) \varpi,
$$

where $\theta_{0}$ is the coordinate at reference time $t_{0}$ and $F_{\theta}(t, \alpha, \delta)$ is a known function representing the projection of the unit parallax ellipse in the measurement direction, i.e. along $\theta$. Note that $F_{\theta}$ depends on the position of the star as well as on time because of the varying shape and phase of the parallax ellipse across the celestial sphere.

From a series of accurate positional measurements, covering $\theta(t)$ over the course of at least one year, it is in principle trivial to determine the three model parameters $\theta_{0}, \mu$ and $\varpi$ by least-squares fitting of Eq. (1.1) to the data. Fitting the measurements in orthogonal directions (e.g., in $\alpha$ and $\delta$ ) gives independent values for $\theta_{0}$ and $\mu$ in each direction, while $\varpi$ should ideally be the same. F. W. Bessel is credited with having made the first reliable determination of a stellar parallax in 1837-1838. His measurements (Bessel 1838) are plotted in Fig. 1, together with a fit of Eq. (1.1). Bessel measured the angular distances of the fifth-magnitude visual double star 61 Cyg from two fainter (magnitude 8-9) reference stars, called ' $a$ ' and ' $b$ ' in his paper. As it happens, the stars form an almost right-angled triangle (see the middle panel in Fig. 1), and the two measurement sets are therefore nearly orthogonal. Fitting Eq. (1.1) separately to each set gives $\varpi=369 \pm 28$ mas 

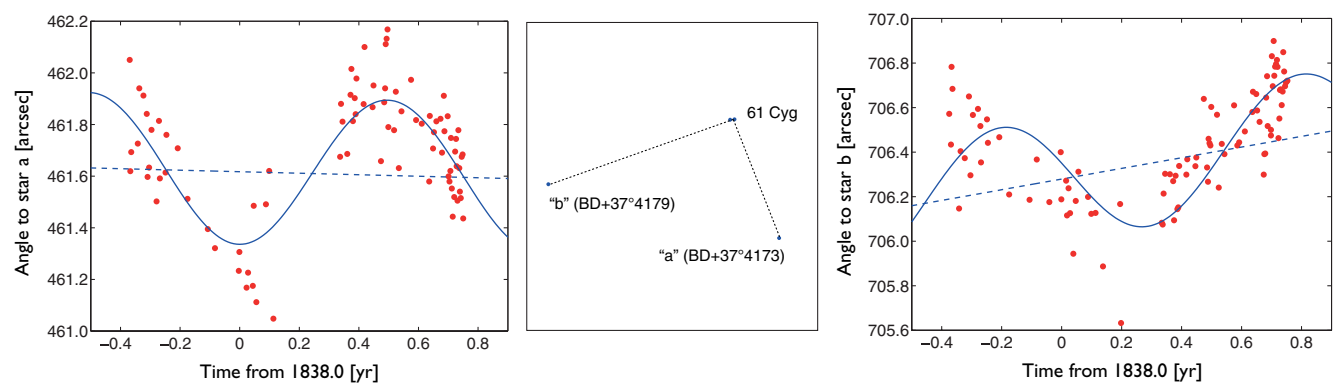

Figure 1. Determination of the parallax of $61 \mathrm{Cyg}$ (data from Bessel 1838). The dots are Bessel's measurements of the angular separations of 61 Cyg from the two reference stars ' $a$ ' and 'b', with the main part of the proper motion subtracted; the solid curves are the fitted model in Eq. (1.1) for a parallax of 314 mas. The dashed lines show the proper-motion part of the model. The middle panel shows the configuration of the stars (North up).

(relative to 'a') and $261 \pm 28$ mas (relative to 'b'), while a simultaneous solution yields $\varpi=314 \pm 20$ mas. These standard errors, computed in the usual manner from the rms residuals, agree perfectly with the mittleren Fehler given by Bessel.

Bessel's observations and analysis illustrate not only the general principle of parallax determination, but also some of its potential difficulties. Nearly 200 measures made over 14 months provided a high degree of redundancy, good coverage of the parallax ellipse, clear separation of proper motion from parallax and sufficient weight for a statistically significant result. The reasonably consistent parallaxes obtained in orthogonal directions gave further confidence in the result. However, since the reference stars are not at infinite distances, the calculated parallaxes are in fact relative: the measured quantities are $\varpi-$ $\varpi_{\mathrm{a}}$ and $\varpi-\varpi_{\mathrm{b}}$. This is because the parallax factor $F_{\theta}$ in Eq. (1.1) at a given time is virtually the same for every star in a small field. Bessel was fortunate in that the reference stars are probably distant giants (judging from their small proper motions), so the required corrections are probably only a few mas. The correction from relative to absolute values is, however, an important limitation for high-precision, small-field parallaxes (cf. Sect. 3.1).

\section{Ground-based parallaxes}

After the pioneering work by Bessel, Struve, and others, progress in stellar trigonometric parallax work was initially very slow - around the year 1900, less than 100 stars had had their parallaxes measured, typically with a standard error of roughly 35 mas (Jackson 1956). The situation improved with the general introduction of photographic techniques and systematic programmes undertaken by a number of observatories. The first General Catalogue compiled by Schlesinger et al. (1924) listed trigonometric parallaxes for 1682 stars; subsequent editions (Schlesinger \& Jenkins 1935; Jenkins 1952, 1963) increased this number to 6390. However, with a typical mean error of 16 mas (Hertzsprung 1952), most of these parallaxes were only marginally more precise than Bessel's value for 61 Cyg. Substantial improvements were made in the 1960s and 70s through the use of dedicated reflectors, in particular the USNO $1.55 \mathrm{~m}$ astrometric (Strand) reflector (Harrington \& Dahn 1980), and by employing automatic measuring engines (microdensitometers) together with digital image-centring techniques (Auer \& van Altena 1978). Standard errors of 4 mas were now consistently obtained also for much fainter stars. The fourth edition of the General Catalogue (van Altena et al. 1995), summarizing the pre-Hipparcos status of the field, contains parallaxes for 8112 stars. 


\subsection{Optical and near-infrared observations}

The introduction of CCDs, and other solid-state detectors for near-infrared observations, revolutionized astrometry. Their positional stability, linearity, and large dynamic range practically eliminated many of the problems that had plagued photographic astrometry for decades, in particular the magnitude-dependent systematic errors. High sensitivity and simple data acquisition increased the efficiency of observations, while near-infrared capability meant that new types of objects became accessible. Parallax programmes in the last decades have tended to focus on nearby $(\lesssim 25 \mathrm{pc})$ ultracool (late M, L and T) stars, providing a more complete inventory of the solar neighbourhood. At optical wavelengths $(<1 \mu \mathrm{m})$ the programs at USNO (Harris et al. 2007; Dahn et al. 2008), ESO/NTT (Ducourant et al. 2008), Torino (TOPP; Smart et al. 2010), CTIO (RECONS; Jao et al. 2011), and ESO (PARSEC; Andrei et al. 2011) ought to be mentioned. In the near-infrared regime $(>1 \mu \mathrm{m})$ there are parallax programmes, e.g., at ESO/NTT (Tinney et al. 2003), USNO (Vrba et al. 2004), CAHA (Schilbach et al. 2009), UKIRT (Marocco et al. 2010), and the CFHT (Dupuy \& Liu 2012). Accuracies in the 1-5 mas range are typical, but some programmes, including those at USNO and the CFHT, reach well below 1 mas. A standard error of 0.3 mas appears to be the state of the art at optical wavelengths (Harris et al. 2005).

\subsection{Radio (VLBI)}

Very Long Baseline Interferometry (VLBI) is a powerful technique for astrometric observations of compact radio sources, including many kinds of Galactic objects such as pulsars, masers in star-forming regions and radio-loud binaries. With baselines on the order of $10,000 \mathrm{~km}$, typical wavelengths of a few $\mathrm{cm}$, and a signal-to-noise ratio $\gtrsim 10$, the theoretical precision of a single observation is on the order of $10 \mu$ as (Pradel et al. 2006). In practice, the precision is limited by how well the path-length fluctuations $(c \tau)$ in the ionosphere $\left(c \tau \propto \lambda^{2}\right)$ and troposphere $\left(c \tau \propto \lambda^{0}\right)$ can be modelled or predicted; residual errors are likely to be several $\mathrm{cm}$, corresponding to an angular measurement precision of approximately 1 mas. The phase-referencing technique, pioneered by Shapiro et al. (1979) and further developed by Lestrade et al. (1990), is used to overcome this limitation and to allow observations of much fainter sources (Chatterjee et al. 2004; Pradel et al. 2006; Peterson et al. 2011). The phase of the target source is observed differentially with respect to a radio-bright quasar at an angular separation $\theta$ of less than a few degrees. The precision is then limited by the differential path-length errors, which are smaller than the absolute path-length errors by roughly a factor $\theta$ [rad]. Provided that the reference quasar is sufficiently stable, the method directly yields the absolute parallax and proper motion of the target source (at redshift 0.1 , the parallax is only a few nano-arcsec).

Parallax determinations using phase referencing are carried out by all international VLBI networks (VLBA, EVN, VERA, LBA), using frequencies typically ranging from 1.4 to $43 \mathrm{GHz}$. A few dozen radio-emitting stars have radio parallaxes with accuracies in the sub-mas range, including Algol, Cyg X-1, the G5 giant HD199178, and several RS CVn binaries and stars in the Orion Nebula Cluster (Lestrade et al. 1999; Menten et al. 2007; Reid et al. 2011; Shapiro et al. 2012). As an example, Ratner et al. (2012) determine a parallax of $10.37 \pm 0.07$ mas for the RS CVn binary IM Peg, in perfect agreement with the Hipparcos value (10.33 \pm 0.76 mas), but with ten times smaller uncertainty. Parallaxes and annual proper motions of pulsars, highly interesting for the study of neutron-star birthplaces and evolution, have been measured with uncertainties down to 0.02 mas (Brisken et al. 2002; Chatterjee et al. 2009; Deller et al. 2009). These are continuum measurements of non-thermal emission. In star-forming regions and circumstellar envelopes of cool giants, maser lines mainly from methanol (at 6.7 and $12.2 \mathrm{GHz}$ ), water 
$(22 \mathrm{GHz})$ and silicon monoxide $(43 \mathrm{GHz})$ provide excellent targets for mapping Galactic structure and kinematics (e.g., Brunthaler et al. 2011; Nyu et al. 2011). A well-known result is the parallax of the star-forming region Sgr B2 close to the Galactic Centre, $\varpi=0.129 \pm 0.012$ mas (Reid et al. 2009b), providing the first trigonometric estimate of $\mathrm{R}_{0}$. Some of the most precise determinations for star-forming regions published to date are $\varpi=0.189 \pm 0.008$ mas for S269 (Honma et al. 2007), $0.476 \pm 0.006$ mas for S252 (Reid et al. 2009a) and 0.199 \pm 0.007 mas for G48.61+0.02 (Nagayama et al. 2011). Thus, the state-of-the-art precision is an impressive 0.01 mas or better. However, some caution should be exercised when interpreting these results, considering that they are usually based on observations at very few epochs and that the maser sources may have quite complex and variable structures (cf. Sect. 4; Miyoshi et al. 2012).

\section{Parallaxes from space}

In the absence of atmospheric disturbances, the precision of optical astrometric measurements is in theory limited only by diffraction and photon noise (Lindegren 2010). Using a telescope of modest $(\sim 1 \mathrm{~m})$ size, wide-band CCD detection, and a $1 \mathrm{~min}$ exposure time, the limiting precision is approximately $5 \mu$ as for a $V=10$ mag star. The main challenges are to provide an instrument of sufficient stability and the means to calibrate it to matching accuracy. This will benefit from weightlessness and the (potentially) stable thermal environment in space, as well as the possibility to cover the whole celestial sphere with a single instrument.

\subsection{Relative parallaxes: Hubble Space Telescope}

The Fine Guidance Sensors (FGS) of the NASA/ESA Hubble Space Telescope (HST) are two-axis shearing interferometers operating in three separate 'pickle'-shaped areas in the outer parts of the HST field of view (Bradley et al. 1991). While two of the interferometers are phase-locked on guide stars, the third (FGS3 and, since 1999, FGS1r) can be used for relative astrometry within the remaining $\sim 15 \times 3 \operatorname{arcmin}^{2}$ pickle. The highly non-trivial astrometric calibration (cf. Benedict et al. 1999) yields an accuracy of approximately 1 mas per observation for the magnitude range $V=3$ to 17 . The HST parallax programme includes a number of Cepheids and RR Lyrae stars for the calibration of their period-luminosity relations, selected Hyades and Pleiades stars, and some fainter stars (e.g., Benedict et al. 2000, 2002, 2007, 2011; Soderblom et al. 2005; McArthur et al. 2011). The typical parallax accuracy is $0.1-0.2$ mas.

As the angular measurements in the FGS are made relative to reference stars that have essentially the same parallax factor $-F_{\theta}$ in Eq. (1.1) - as the target star, owing to the relatively small field, the measured parallaxes are relative and it is necessary to add the estimated mean parallax of the reference stars. This correction is typically $0.8-1.5$ mas with an uncertainty of at least $10 \%$ (see references above).

\subsection{Absolute parallaxes: Hipparcos and Gaia}

The Hipparcos satellite, launched in 1989, introduced a completely new technique for absolute parallax determination based on large-angle optical measurements (Lindegren \& Bastian 2011). By projecting two widely ( 1 rad) separated fields in the same focal plane, angular measurements could be made between stars with very different parallax factors $\left(F_{\theta}\right)$. By combining such measurements made at different times and in different geometric configurations it was possible to solve the absolute parallaxes of all stars without any a priori assumption about their values. The Hipparcos parallax zero point has been questioned mainly on the basis of the discrepant mean parallax obtained for the Pleiades 

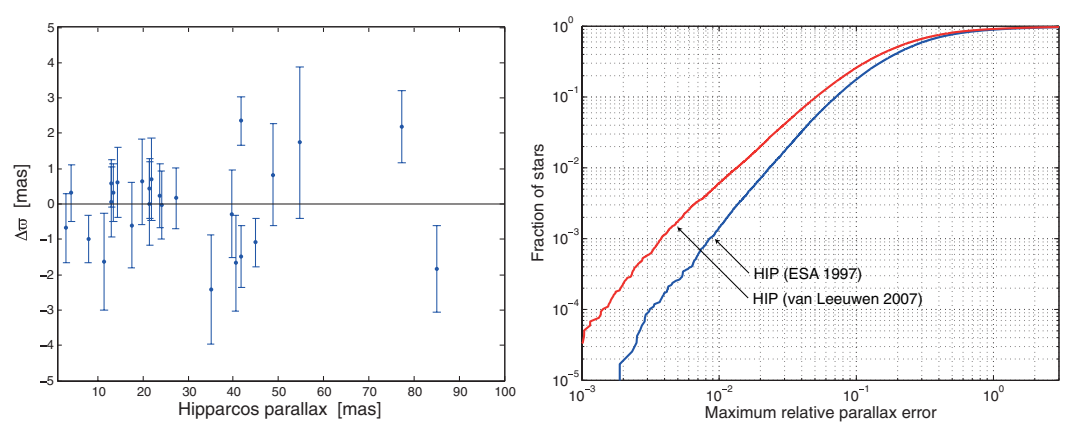

Figure 2. (left) Comparison of Hipparcos parallaxes (ESA 1997) with the 26 orbital parallaxes compiled by Tomkin (2005). $\Delta \varpi=\varpi_{\text {HIP }}-\varpi_{\text {orb }}$ is plotted versus $\varpi_{\text {HIP }}$, with error bars representing the combined standard errors. The weighted mean difference is $\langle\Delta \varpi\rangle=+0.05 \pm 0.19$ mas. (right) Cumulative distribution of the relative parallax uncertainty $\sigma_{\varpi} / \varpi$ for the original Hipparcos Catalogue (ESA 1997) and for the re-reduction by van Leeuwen (2007).

cluster (e.g., Pinsonneault et al. 1998; Soderblom et al. 2005), a controversy that has not yet been fully resolved (see Perryman 2009 for a detailed discussion). While it is possible that spatial correlations can account for 'systematics' seen on a small scale (a few degrees), it is believed that the zero point is essentially correct on a global scale, as suggested by several tests including comparisons with ground-based parallaxes (Harris et al. 1997), HST/FGS results (McArthur et al. 2011), orbital parallaxes for binaries (Tomkin 2005; Fig. 2, left), and distances derived from asteroseismology coupled with the infrared flux method (Silva Aguirre et al. 2012).

The Hipparcos Catalogue (ESA 1997) gives parallaxes for some 118,000 stars, including all stars brighter than a predefined survey limit $(V \simeq 8 \mathrm{mag})$ and a selection of fainter stars. Typical standard errors are 0.8-2 mas. The many astrophysical applications of these data have been extensively reviewed by Perryman (2009).

The new reduction of the raw Hipparcos data by van Leeuwen (2007) resulted in significantly improved parallaxes for most stars, and especially for the bright ones, mainly thanks to much more elaborate modelling of the satellite's attitude. Standard errors down to 0.2 mas are claimed for bright stars $(V<5 \mathrm{mag})$, while the overall median standard error is reduced from 1.1 mas in the original catalogue to 0.9 mas in the re-reduction. Fig. 2 (right) shows the improvement in terms of the distribution of the relative parallax errors (or, equivalently, the relative distance errors). The improvement is most dramatic for the smallest relative errors; for example, the fraction of stars with $\sigma_{\varpi} / \varpi \leqslant 0.01$ increased from $0.14 \%$ (168 stars) to $0.61 \%$ (717 stars).

ESA's Gaia satellite is based on the same general principles as Hipparcos, but will employ much more efficient detectors (CCDs) and larger telescopes to achieve parallax accuracies in the 0.01-0.3 mas range for roughly one billion stars in the magnitude range from 6 to 20. For a recent summary of the science objectives, instrument description, and science performance, see de Bruijne (2012). With an expected launch in 2013 and a nominal lifetime of five years, the final catalogue will be available in 2021. High-precision trigonometric distances (better than 1\%) will be obtained for millions of stars. Although most of the latter will be nearby dwarfs, the entire Hertzsprung-Russell diagram will be very well sampled, including many rare objects and stars in rapid evolutionary phases. The parallaxes will be absolute, based on the wide-angle measurement principle described above. This places very stringent demands on the stability of the instrument and especially of the basic angle between the two viewing directions. Passive stabilization of the instrument guarantees that the global parallax zero point error is less than $3.5 \mu \mathrm{as}$, but 

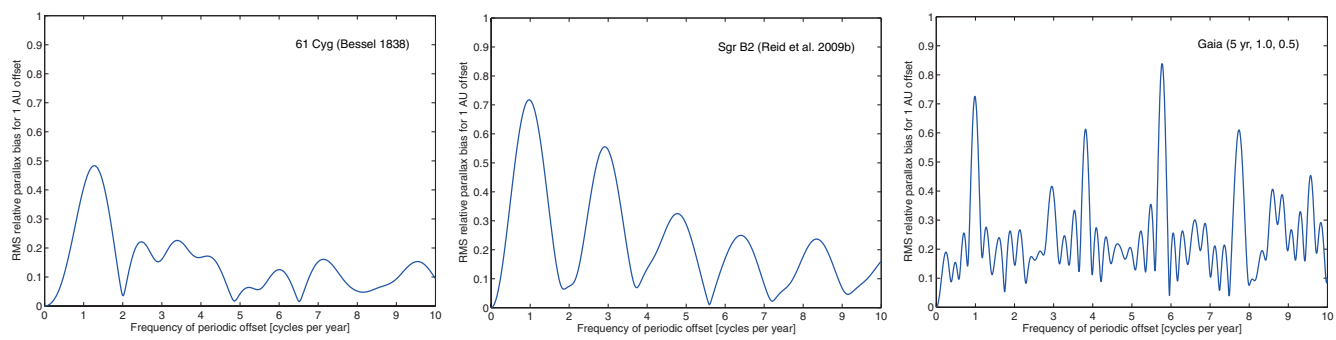

Figure 3. Three examples of the Parallax Sensitivity Spectrum (see text). From left to right, they refer to the actual observations of 61 Cyg by Bessel (1838), of Sgr B2 by Reid et al. (2009b) and Gaia's projected observations in $2014.0-2019.0$ of a star at position $(\alpha, \delta)=(1.0,0.5)$ rad.

in addition the interferometric Basic Angle Monitor (Meijer et al. 2008) will allow elimination of much smaller variations in the data analysis, reducing the zero point error to below $0.1 \mu$ as. Statistically, the Gaia parallaxes should therefore be meaningful even at extragalactic distances.

\section{Limitations: Source structure}

Of the various fundamental and practical limitations to the parallax method, some of which have been briefly mentioned above (atmospheric effects, instrument calibration, correction from relative to absolute values), here I will only discuss the role of source structure as a possible generator of random and systematic errors.

In all kinds of astrometric measurements, the angular precision obtained is many times better than the actual resolution of the instrument used, i.e. the beam size of a radio interferometer, or the point-spread function of an optical telescope. The improvement factor is essentially given by the signal-to-noise ratio of the observation. For an unresolved source, the measured position is the centre of gravity of the brightness distribution, or what in the optical is known as the photocentre. Since most astrometric observations are made for unresolved sources, it is in general their photocentres that matter.

The parallax method depends on accurate measurements of annual angular variations on a scale corresponding to a linear displacement of 1 au at the distance of the source. We therefore need to worry about photocentre variations that occur on spatial scales of $\sim 1$ au (or larger), and on temporal scales of $\sim 1$ yr (or shorter). Dynamically, these would be associated with masses of $\sim 1 \mathrm{M}_{\odot}$ (no coincidence!), and might therefore be quite common in stellar astrophysics. Indeed, a range of phenomena could generate such variations, including binaries with orbital periods of less than a few years, binaries with variable components, supergiants and other objects with extended envelopes, disks, jets, etc. Bastian \& Hefele (2005) give a general overview of the effects, while orbital motion is specifically discussed by Söderhjelm (2005), star spots by Eriksson \& Lindegren (2007) and stellar granulation in supergiants by Svensson \& Ludwig (2005), Pasquato et al. (2011) and Chiavassa et al. (2011).

The sensitivity of parallax measurements to photocentric variability depends in a complex way on the number of measurements, their temporal distribution and the total length of the measurement series. To quantify this dependence I propose to introduce the Parallax Sensitivity Spectrum (PSS), showing the rms expected parallax bias generated by a photocentric wobble of 1 au amplitude, and random phase in each coordinate, as a function of the frequency $f$ of the wobble. The PSS, expressed in units of the true parallax, can be computed for any object when the times of observation are known. Three examples are given in Fig. 3. The first two are for actual observations (of 61 Cyg and Sgr 
B2), the third for a typical sequence of Gaia measurements. A few interesting features are noted in all PSS diagrams. For very low frequencies $\left(f \ll 1 \mathrm{yr}^{-1}\right)$ the PSS goes to zero because the photocentre variation is then completely absorbed by the proper-motion term in Eq. (1.1). Furthermore, there is inevitably a peak around $f=1 \mathrm{yr}^{-1}$ caused by variations that mimic the parallax ellipse (producing a positive or negative bias, depending on the phases). Finally, all peaks have a width of approximately $1 / T$, if $T$ is the total length of the measurement series. The presence of secondary peaks depends strongly on the temporal distribution of the measurements. The rather uniform coverage of $61 \mathrm{Cyg}$ tends to dampen these peaks, while the precession motion of the Gaia spin axis, with a period of 63 days, produces a strong peak at $f=5.8 \mathrm{yr}^{-1}$. For VLBI observations, the tendency to schedule observing sessions only at the extreme points of the parallax ellipse, which is economical in terms precision gained per observing hour, makes the result more sensitive to variability at other frequencies. The PSS thus appears to be a useful tool to characterize possible parallax biases due to variable source structure.

\section{Conclusions}

The past decades have seen tremendous progress in trigonometric distance determinations, with several optical and near-IR techniques reaching accuracies of $0.2-1$ mas, radio interferometry for compact non-thermal sources reaching 0.01 mas, and Gaia promising similar accuracies for millions of stars. It is often claimed that the parallax method is model-independent, but it always involves fitting some simplified model like Eq. (1.1) to measured data, which in turn may have been derived using some complex modelling of the source, atmosphere and instrument. Each technique has its own limitations and a cautious user of the data should be aware of them.

\section{References}

Andrei, A. H., Smart, R. L., Penna, J. L., et al. 2011, AJ, 141, 54

Auer, L. H. \& van Altena, W. F. 1978, AJ, 83, 531

Bastian, U. \& Hefele, H. 2005, in: The Three-Dimensional Universe with Gaia (Turon, C., O'Flaherty, K.S., \& Perryman, M.A.C., eds.), ESA SP-576, p. 215

Benedict, G. F., McArthur, B., Chappell, D. W., et al. 1999, AJ, 118, 1086

Benedict, G. F., McArthur, B. E., Franz, O. G., et al. 2000, AJ, 119, 2382

Benedict, G. F., McArthur, B. E., Forveille, T., et al. 2002, ApJ, 581, L115

Benedict, G. F., McArthur, B. E., Feast, M. W., et al. 2007, AJ, 133, 1810

Benedict, G. F., McArthur, B. E., Feast, M. W., et al. 2011, AJ, 142, 187

Bessel, F. W. 1838, Astron. Nachr., 16, 65

Bradley, A., Abramowicz-Reed, L., Story, D., Benedict, G., \& Jefferys, W. 1991, PASP, 103, 317

Brisken, W. F., Benson, J. M., Goss, W. M., \& Thorsett, S. E. 2002, ApJ, 571, 906

Brunthaler, A., Reid, M. J., Menten, K. M., et al. 2011, Astron. Nachr., 332, 461

Chatterjee, S., Cordes, J. M., Vlemmings, W. H. T., et al. 2004, ApJ, 604, 339

Chatterjee, S., Brisken, W. F., Vlemmings, W. H. T., et al. 2009, ApJ, 698, 250

Chiavassa, A., Pasquato, E., Jorissen, A., et al. 2011, A\&A, 528, A120

Dahn, C. C., Harris, H. C., Levine, S. E., et al. 2008, ApJ, 686, 548

de Bruijne, J. H. J. 2012, Ap\&SS, 341, 31

Deller, A. T., Tingay, S. J., Bailes, M., \& Reynolds, J. E. 2009, ApJ, 701, 1243

Ducourant, C., Teixeira, R., Chauvin, G., et al. 2008, A\&A, 477, L1

Dupuy, T. J. \& Liu, M. C. 2012, ApJS, 201, 19

Eriksson, U. \& Lindegren, L. 2007, A\&A, 476, 1389

ESA 1997, The Hipparcos and Tycho Catalogues, ESA SP-1200

Harrington, R. S. \& Dahn, C. C. 1980, AJ, 85, 454 
Harris, H. C., Dahn, C. C., \& Monet, D. G. 1997, in: Hipparcos - Venice '97 (Bonnet, R., Høg, E., Bernacca, P.L., et al., eds.), ESA SP-402, p. 105

Harris, H. C., Canzian, B., Dahn, C. C., et al. 2005, ASP Conf. Ser., 338, 122

Harris, H. C., Dahn, C. C., Canzian, B., et al. 2007, AJ, 133, 631

Hertzsprung, E. 1952, Observatory, 72, 242

Honma, M., Bushimata, T., Choi, Y. K., et al. 2007, PASJ, 59, 889

Jackson, J. 1956, Vistas in Astronomy, 2, 1018

Jao, W.-C., Henry, T. J., Subasavage, J. P., et al. 2011, AJ, 141, 117

Jenkins, L. F. 1952, General Catalogue of Trigonometric Stellar Parallaxes, Yale Obs.

Jenkins, L. F. 1963, Supplement to the General Catalogue of Trigonometric Stellar Parallaxes, Yale Obs.

Lestrade, J.-F., Rogers, A. E. E., Whitney, A. R., et al. 1990, AJ, 99, 1663

Lestrade, J.-F., Preston, R. A., Jones, et al. 1999, A\&A, 344, 1014

Lindegren, L. 2010, in: Observing Photons in Space (Huber, M. C. E., Pauluhn, A., Culhane, J. L., et al., eds.), ISSI Sci. Rep. Ser., 9, p. 279

Lindegren, L. \& Bastian, U. 2011, EAS Publ. Ser., 45, 109

Marocco, F., Smart, R. L., Jones, H. R. A., et al. 2010, A\&A, 524, A38

McArthur, B. E., Benedict, G. F., Harrison, T. E., \& van Altena, W. 2011, AJ, 141, 172

Meijer, E. A., Nijenhuis, J. N., Vink, R. J. P., \& Kamphues, F. 2008, SPIE Conf. Ser., 7010, $70102 \mathrm{O}$

Menten, K. M., Reid, M. J., Forbrich, J., \& Brunthaler, A. 2007, A\&A, 474, 515

Miyoshi, M., Asaki, Y., Wada, K., \& Imai, H. 2012, New Astron., 17, 553

Nagayama, T., Omodaka, T., Handa, T., et al. 2011, PASJ, 63, 719

Nyu, D., Nakagawa, A., Matsui, et al. 2011, PASJ, 63, 63

Pasquato, E., Pourbaix, D., \& Jorissen, A. 2011, A\&A, 532, A13

Perryman, M. 2009, Astronomical Applications of Astrometry: Ten Years of Exploitation of the Hipparcos Satellite Data, Cambridge Univ. Press

Peterson, W. M., Mutel, R. L., Lestrade, J.-F., Güdel, M., \& Goss, W. M. 2011, ApJ, 737, 104

Pinsonneault, M. H., Stauffer, J., Soderblom, D. R., King, J. R., \& Hanson, R. B. 1998, ApJ, 504,170

Pradel, N., Charlot, P., \& Lestrade, J.-F. 2006, A\&A, 452, 1099

Ratner, M. I., Bartel, N., Bietenholz, M. F., et al. 2012, ApJS, 201, 5

Reid, M. J., Menten, K. M., Brunthaler, A., et al. 2009a, ApJ, 693, 397

Reid, M. J., Menten, K. M., Zheng, X. W., Brunthaler, A., \& Xu, Y. 2009b, ApJ, 705, 1548

Reid, M. J., McClintock, J. E., Narayan, R., et al. 2011, ApJ, 742, 83

Schilbach, E., Röser, S., \& Scholz, R.-D. 2009, A\&A, 493, L27

Schlesinger, F., Palmer, M., \& Pond, A. 1924, General Catalogue of Stellar Parallaxes, Yale Obs.

Schlesinger, F. \& Jenkins, L. F. 1935, General Catalogue of Stellar Parallaxes, $2^{\text {nd }}$ ed., Yale Obs.

Shapiro, I. I., Wittels, J. J., Counselman, III, C. C., et al. 1979, AJ, 84, 1459

Shapiro, I. I., Bartel, N., Bietenholz, M. F., et al. 2012, ApJS, 201, 1

Silva Aguirre, V., Casagrande, L., Basu, S., et al. 2012, ApJ, 757, 99

Smart, R. L., Ioannidis, G., Jones, H. R. A., Bucciarelli, B., \& Lattanzi, M. G. 2010, A\&A, 514, A 84

Soderblom, D. R., Nelan, E., Benedict, G. F., et al. 2005, AJ, 129, 1616

Söderhjelm, S. 2005, in: The Three-Dimensional Universe with Gaia (Turon, C., O'Flaherty, K.S., \& Perryman, M.A.C., eds.), ESA SP-576, p. 97

Svensson, F. \& Ludwig, H.-G. 2005, in: $13^{\text {th }}$ Cambridge Workshop on Cool Stars, Stellar Systems and the Sun (Favata, F., Hussain, G.A.J., \& Battrick, B., eds.), ESA SP-560, p. 979

Tinney, C. G., Burgasser, A. J., \& Kirkpatrick, J. D. 2003, AJ, 126, 975

Tomkin, J. 2005, ASP Conf. Ser., 336, 199

van Altena, W. F., Lee, J. T., \& Hoffleit, E. D. 1995, The General Catalogue of Trigonometric Stellar Parallaxes, $4^{\text {th }}$ ed., Yale Obs.

van Leeuwen, F. 2007, Hipparcos, the New Reduction of the Raw Data, Astrophys. Space Sci. Lib., vol. 350

Vrba, F. J., Henden, A. A., Luginbuhl, C. B., et al. 2004, AJ, 127, 2948 